\title{
MÉTODO DEL AULA INVERTIDA UTILIZANDO LA MASCOTA, EN LA ESCUELA RURAL
}

\section{FLIPPED CLASSROOM METHOD USING A PET, IN RURAL SCHOOL}

\author{
Freddy Urley Urón ${ }^{1}$ \\ Luisa Stella Paz Montes² \\ Mawency Vergel Ortega ${ }^{3}$ \\ UFPS
}

\section{RESUMEN}

La investigación tiene como objetivo diseñar una herramienta didáctica que ayude a los estudiantes del sector rural a comprender las nociones y elementos básicos de la geometría de una manera creativa, sencilla e innovadora utilizando los recursos del entorno como son las mascotas y aportar con esta experiencia un cambio significativo en los demás compañeros y

1 Universidad Francisco de Paula Santander, Norte de Santander, Cúcuta, Colombia, correo: furon66@hotmail. com Orcid: https://orcid.org/0000-0003-4942-283X

2 Universidad Francisco de Paula Santander, Norte de Santander, Cúcuta, Colombia

Correo: luisastellapm@ufps.edu.co, Ipazmontes@ gmail.com orcid: https://orcid.org/0000-0002-8887-3441

3 Universidad Francisco de Paula Santander, Norte de Santander, Cúcuta, Colombia. Correo: mawencyvergel@ ufps.edu.co, orcid: https://orcid.org/0000-0001-8285-2968 maestros del Modelo Escuela Nueva. El método de enseñanza del aula invertida, en el que los estudiantes utilizan fuentes proporcionadas por sus profesores para aprender antes de clase, y el tiempo de la clase se utiliza para la cooperación grupal y para resolver problemas e inquietudes presentadas sobre los temas, en forma conjunta. Se enmarcó dentro del enfoque cualitativo, con apoyo cuantitativo, se desarrolló bajo el método de investigación acción, técnicas de recolección de información fueron: observación directa, entrevista estructurada, test y encuesta. En la fase uno la población objeto de estudio está constituida por 11 estudiantes de grado quinto de la Escuela nueva Sede el Hoyo de municipio El Carmen, Norte De Santander y el 1 docente y la validez estuvo constituida por una muestra de 150 estudiantes de la zona rural y urbana de Cúcuta. Resultados permiten apreciar que si el estudiante adquiere la fundamentación teórica y 
aplica lo aprendido en el aula de clase, mejora conceptualización, así mismo, la estrategia didáctica a través del perro mejora la motivación, generación de ideas y rendimiento de os estudiantes. Se concluye que a través de la interacción con un canino es posible desarrollar temas de geometría lineal, diferencial, cálculo, convirtiéndose en una experiencia creativa, dinámica y enriquecedora.

PALABRAS CLAVE: Enseñanza, geometría, mascota, aula invertida, cálculo

\section{ABSTRACT.}

The objective of the research is to design a didactic tool that helps students in the rural sector to understand the basic notions and elements of geometry in a creative, simple and innovative way using the resources of the environment such as pets, and to contribute with this experience a significant change in the other classmates and teachers of the Escuela Nueva Model. The inverted classroom teaching method, in which students use sources provided by their teachers to learn before class, and class time is used for group cooperation and to solve problems and concerns presented on the topics, jointly. It was framed within the qualitative approach, with quantitative support, it was developed under the action research method, data collection techniques were: direct observation, structured interview, test and survey. In phase one, the study population consisted of 11 fifth grade students from the Escuela Nueva Sede EI Hoyo in the municipality of El Carmen, Norte de Santander, and 1 teacher, and the validity was constituted by a sample of 150 students from the rural and urban areas of Cúcuta. Results show that if the student acquires the theoretical foundation and applies what he/she has learned in the classroom, he/she improves conceptualization. The didactic strategy through the dog improves the motivation, generation of ideas and performance of the students. It is concluded that through the interaction with a canine it is possible to develop topics of linear geometry, differential geometry, calculus, becoming a creative, dynamic and enriching experience.

Keywords: Teaching, geometry, pet, flipped classroom, calculus

\section{INTRODUCCIÓN}

La geometría siempre ha estado presente en el aprendizaje de las matemáticas, ha generado creatividad y permitido tener una interdisciplinariedad con otras áreas de la formación escolar, con el objeto de rescatar su instrucción en las aulas de clase, se ve la necesidad de diseñar formular una propuesta que se encamine a la enseñanza de las diferentes figuras geométricas y enriquecer el pensamiento matemático y espacial a través de la relación entre el estudiante y su mascota, proponiendo actividades lúdicas que permitan transformar el proceso de enseñanzaaprendizaje de la Geometría en las escuelas primarias rurales multigrado ( Escuela Nueva).

Por lo anterior es necesario hacer cambios en el orden didáctico-metodológico, que permita tener conocimiento del entorno y el contexto escolar rural en el que conviven los estudiantes y sus familias, para una eficaz aplicación de los conceptos adquiridos, donde los estudiantes perciban un aprendizaje activo y real, al involucrar los temas aprendidos en el aula de clases en las actividades de su finca y ver de una forma practica el uso de la geometría en su quehacer cotidiano.

El objetivo es brindar una estrategia propicia de una etapa de transformación en el orden pedagógico y didáctico-metodológico, al permitir un trabajo colaborativo en el proceso y poder apreciar en toda su dimensión la aplicación de la geometría en todas las situaciones en que nos encontramos inmersos en nuestro entorno 
rural y la importancia en contexto educativo asociando conceptos y medidas.

\section{MARCO TEÓRICO}

\section{1 aprendizaje de la geometría en el contexto} rural.

La enseñanza de la geometría en la básica primaria es de suma importancia en el valioso y fundamental proceso del aprendizaje de las matemáticas, ya que mediante la geometría se fortalece la creatividad, el arte y las habilidades intelectuales en los niños.

La enseñanza de la geometría debe ser un eje central en el currículo escolar, por ser una disciplina de carácter creativo, imaginativo y formativo, que permite desarrollar el razonamiento matemático en nuestros estudiantes. La adquisición y mecanización de los conceptos y las relaciones geométricas cobran importancia en la resolución problemas de nuestra cotidianidad en las diversas situaciones del ambiente social que se afrontan en el día a día. La construcción de los conocimientos geométricos se logra gracias a la interacción entre el sujeto y los objetos, se da cuando el estudiante esta frente a un problema, tratando de darle solución, utilizando conceptos adquiridos, poniéndolos a prueba y/o modificándolos.

Es importante que el estudiante comprenda la utilidad de las matemáticas en la vida real, y en especial dela geometría, porque son conocimientos que no se aprenden estudiando de memoria, sino que se tienen que entender de una forma práctica y tangible, lo cual hace que el proceso enseñanza - aprendizaje, sea dispendioso.

El aprendizaje que tiene lugar en los niños es significativo y de gran relevancia, además de estar ligado al pensamiento y a la experiencia. La adquisición de conceptos y contenidos conlleva en los alumnos una observación, exploración y una experimentación de la realidad. El entender todo ello dará lugar al aprendizaje. Este aprendizaje se elabora de forma gradual, ordenada y se tienen en cuenta dos procesos: la asimilación de los componentes proyectivos y métricos del espacio, y la adquisición de las formas geométricas.

Desafortunadamente la geometría siendo un área tan importante no se orienta con la intensidad horaria que merece, su enseñanza y aprendizaje se limita a muy pocas horas en el aula de clases, desconociendo que estamos rodeados de formas y figuras geométricas $y$ donde no se le da al alumno la oportunidad de explorar las formas, colores, el arte y el diseño, explorar su entorno, utilizar sus espacios y sus medidas, el desarrollo de sus habilidades que permitan la asimilación y el aprendizaje significativo en los estudiantes, donde él pueda desarrollar mediante una metodología activa sus propios razonamientos.

Existen múltiples factores que interfieren en el desarrollo del pensamiento espacial, algunos de éstos se encuentran reflejados en las dificultades y los problemas que presentan los estudiantes sobre la comprensión de los temas geométricos, estos son relacionados al tipo de enseñanza que han recibido en clase y en errores en que los docentes incurren al hacer que el proceso educativo se base en la memorización de definiciones, teoremas y propiedades de manera mecánica, descontextualizada y aislada de otras áreas del saber aislando al estudiante de la interdisciplinariedad con otras áreas del conocimiento.

En este sentido, Godino J (2003) afirman que: Los estudiantes aprenden matemáticas por medio de las experiencias que les proporcionan los profesores. Por tanto, la comprensión de las matemáticas por parte de los estudiantes, su capacidad para usarlas en la resolución de problemas, y su confianza y buena disposición 
hacia las matemáticas están condicionadas por la enseñanza que encuentran en la escuela.

La realidad que viven las instituciones educativas rurales, muestra que el desarrollo y desinterés en el pensamiento espacial, no es tan importante como otros tipos de pensamientos, puesto que la clase de geometría en muchas ocasiones es reemplazada por actividades propias de otras áreas y la metodología que el maestro aplica en su enseñanza es basada en la transmisión de conocimientos mediante el dictado y copia de definiciones, lo que ha ocasionado que los estudiantes no hayan desarrollado las competencias geométrico espaciales respectivas, ni las articulan con otros saberes matemáticos, lo cual resulta fundamental para avanzar a niveles más formales del conocimiento geométrico.

Es por eso que se reconoce la necesidad educativa de diseñar y aplicar actividades pedagógicas innovadoras que permitan la optimización de la clase de geometría, a través de los contenidos geométricos dentro del aula de clase, utilizando los recursos del entorno social y familiar del alumno; por tal razón se pretende trabajar la parte de la geometría con un animal doméstico, ya que los niños y los animales domésticos se interrelacionan constantemente, por lo tanto, surge la necesidad, de cambiar el paradigma de la enseñanza-aprendizaje tradicional de las matemáticas, en donde se hace énfasis en la memorización sin sentido de conceptos y propiedades para buscar nuevos modelos pedagógicos, que posibiliten la aplicación de estrategias didácticas en las que el aprendizaje de la geometría sea significativo y perdurable en el tiempo.

\subsection{Las mascotas como instrumento de aprendizaje}

Los conceptos geométricos, en muchas ocasiones son difíciles de comprender debido a que no se les muestra la aplicación ni se relaciona con el entorno rural donde viven; por lo tanto es imperante relacionar una rama de la matemática, como lo es la geometría plana con el medio ambiente, a través de la observación de los animales del entorno donde se convive, de manera específica con las mascotas,

En el sector rural la tenencia de mascotas es muy común, siendo una de las más utilizadas por los habitantes, el perro, mascota que se puede convertir en una herramienta pedagógica muy valiosa e interesante para la comprensión de conceptos geométricos.

¿ por qué un perro como una herramienta en el aprendizaje?, es una explicación sencilla, en cada hogar rural siempre está presente como parte de la familia, esa mascota fiel, de fácil cuidado, noble y fácil de llevar al aula de clase, mascota que tiene diversidad de formas de acuerdo a su raza, tamaño y demás características que conforman su anatomía; la mayoría de los alumnos, en el área rural, y los animales domésticos se interrelacionan constantemente, y por tal razón es muy sencillo que el estudiante pueda palpar su mascota y poco a poco descubrir las figuras geométricas que conforman la anatomía del perro y poder dibujar, medir e identificar que todo lo que nos rodea es geométrico y medible, además de incentivar el cuidado y respeto hacia los animales.

Es muy importante que el alumno desde el inicio de sus clases en la básica primaria comience a manejar la geometría de una manera activa, real, manipulando y conociendo su entorno, transformando desde su perspectiva el mundo donde vive, creando formas, figura, trazos y dibujos donde el plasme su imaginación. Por eso al trabajar con la mascota (perro) en el aula de clases el niño se desinhibe, se siente motivado y curioso frente a esta herramienta innovadora y a su vez extraña, comenzará a preguntarse ¿un perro me puede enseñar a trabajar en geometría?, y al comenzar a encontrar esas 
figuras ocultas; comienza una etapa exploratoria y permanece atento a cada descubrimiento y forma encontrada, empieza a ver a su mascota desde una parte más humana, generando valores y fomentando su actitud artística.

\subsection{Educación asistida con perros}

De otra parte, la vinculación de animales en el entorno de los hogares y específicamente del perro como mascota y en la ayuda de algunos quehaceres ha sido fundamental históricamente y se han establecido vínculos que pueden ser aprovechados en los procesos de aprendizaje. Andrew \& Edney (1995) señalan que "con el sometimiento del perro, los humanos primitivos forjaron probablemente uno de los eslabones de la cadena de su propia civilización"

En este sentido Correa C (2019) señala que: La integración de estrategias educativas asistidas con perros en entornos escolares evidencia beneficios en los estudiantes en cuanto al aumento de autoestima, motivación, seguridad en el ambiente escolar, interés por la lectura, socialización, mejoramiento del humor, memoria de trabajo, cooperación y disminución de sentimientos negativos como la depresión, ansiedad, aburrimiento y soledad; además de la eliminación de barreras sociales, de conductas desadaptativas en el aula y la disminución de comportamientos manipuladores por parte de los niños(as) y jóvenes. Lo que nos permite integrar los conceptos correspondientes a geometría mediante la utilización de dicha mascota.

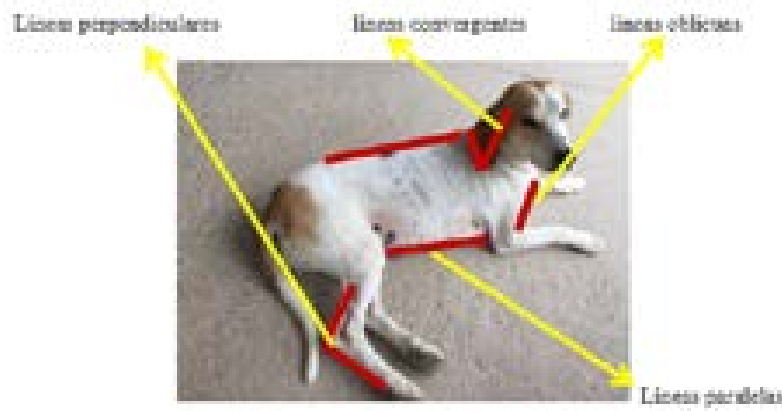

Figura 1. Líneas. Fuente: Urón (2020)

\subsection{Enfoque flipped classroom. Estrategia del aula invertida.}

El enfoque Flipped Classroom apareció inicialmente a principios del siglo XIX. La Academia Militar de los Estados Unidos en West Point creó un conjunto de métodos de enseñanza en el que los estudiantes utilizaban fuentes proporcionadas por sus profesores para aprender antes de clase, mientras que el tiempo de clase se utilizaba para la cooperación grupal para resolver problemas en forma conjunta. Este método de enseñanza refleja perfectamente el concepto básico que subyace en Flipped Classroom.

Lage, Platt y Treglia (2000) introdujeron un "nuevo" método de enseñanza mientras enseñaban en la Universidad de Miami. En sus clases, el multimedia y la web se utilizaban para alentar a los estudiantes a ver videos de aprendizaje en casa, seguidos de un trabajo cooperativo grupal en el aula. Este método de enseñanza era básicamente una versión rudimentaria del Flipped Classroom, pero ese término específico no se había acuñado para ese formato de enseñanza en ese momento. En 2001, el Instituto de Tecnología de Massachusetts desarrolló 'proyectos de cursos abiertos' enfocados como Recursos Educativos Abiertos (REA) que sentaron las bases para la aplicación de un modelo de FC (Flipped Classroom). En 2004, Salman Khan hizo videos de materiales de formación y los subió a un sitio web, que pronto se hizo muy popular entre los estudiantes. Más tarde, fundó Khan Research Institution y subió aún más materiales de aprendizaje a la red, lo que impulsó el rápido desarrollo del FC.

El enfoque de aula invertida implica un mayor grado de responsabilidad para los estudiantes. Esto, acompañado del grado de automotivación que se desarrolle. La implementación de esta técnica persigue una doble finalidad: una es involucrar a los estudiantes en realizar actividades previas a la clase teórica y en 
segundo lugar instaurar un cambio de mentalidad en los docentes de tal manera que pasen más tiempo interactuando con los estudiantes en el lugar de realizar exposiciones eminentemente teóricas.

"La misión principal de la escuela, ya no es enseñar cosas" (Tonucci, 2008); es decir, lo importante ya no es la instrucción, por muy personalizada que esta sea. Es necesario construir procesos educativos; a partir de la comunicación entre actores consciente del alumnado; y a partir de un profesorado que sepa guiar y estimular, el cual ya no puede seguir siendo el protagonista, entre otros motivos, porque es muy complicado serlo en un aula multigrado. El modelo de clase invertida, por lo tanto, combina la instrucción directa y la generación de dinámicas de aprendizaje activo.

El modelo de aula invertida o denominada Flipped Classroom (en inglés), La citada práctica educativa, con base en Sams \& Bergmann (2012), es un modelo pedagógico que consiste en invertir los dos momentos que intervienen en la educación tradicional: el primer momento que corresponde a las actividades propias de la clase como la exposición de los contenidos por parte del docente y, el segundo, a la realización de las actividades fuera de la escuela, como las tareas.

Es así como en el modelo aula invertida las tareas o proyectos se concretan en el salón de clase y los contenidos temáticos son aprendidos fuera de la escuela (Gómez, Rojas, Vergel, 2021). De esta forma, la distribución del tiempo es reorganizada, tanto dentro como fuera de clase, para que el estudiante pueda cumplir con los otros deberes escolares y las otras áreas de aprendizaje obligatorio en el plan de área, es importante recalcar que el estudiante es el responsable de su propio aprendizaje (Maldonado, Vergel, Rojas, 2021). En consecuencia, la clase se dedica a un aprendizaje basado en proyectos, más activo, de alto procesamiento cognitivo, donde los estudiantes trabajan juntos para resolver los problemas de su entorno, locales o globales $-\mathrm{u}$ otras aplicaciones del mundo real- para obtener una comprensión más profunda del tema.

El modelo de clase invertida se ha caracterizado por su flexibilidad y versatilidad, pero no se debe tomar como un cambio de metodología, es en realidad un cambio de la rutina de clase y una modificación a la explicación del tema en clase y las actividades en casa, y lo más interesante un cambio en la escuela, ya que se va transformando poco a poco en un laboratorio de cambio de conceptos entre el alumno - maestro, el maestro - el alumno y alumno-alumno.

\section{METODOLOGÍA}

La presente investigación es de carácter cualitativo con apoyo cuantitativo, según Hernández, Fernández y Baptista (2010) "La investigación cualitativa se enfoca en comprender los fenómenos, explorándolos desde la perspectiva de los participantes en un ambiente natural y en relación con su contexto". Teniendo en cuenta que se pretende indagar sobre la esencia de las experiencias respecto a un fenómeno o proceso se abordara mediante un diseño fenomenológico. La población objeto de estudio inicial para construir la propuesta estuvo constituida por los 11 estudiantes de grado quinto de la Escuela nueva Sede el Hoyo de municipio El Carmen, Norte De Santander y 1 docente, con los cuales se realizará la investigación.

Se basó en el método de investigación acción participante, como estrategia metodológica que busca involucrar y sensibilizar a una comunidad mediante el conocimiento y la solución de problemas; con el fin de generar una transformación social desde un interés emancipador. Se aplicó siguiendo los cuatro momentos o fases recomendadas por Latorre 
(2003): planificación, acción, observación y reflexión.

En torno a cuantitativo para validar la estrategia didáctica, se aplica fase dos realizando pretest y postest a una muestra por conglomerado de 150 estudiantes de Cúcuta de los grados quinto, séptimo, décimo. Las variables fueron expresadas con media aritmética y desviación estándar. Las variables del cuestionario cuyo alfa de Conbrach fue de 0.89 e índice de kappa $\mathrm{K}=0.91$ considerada optima (Méndez, Moreno, Vergel, 2020); para su comparación se utilizó estadístico chi-cuadrado $X^{2}$, considerando como valor de significación estadística una $p<0.05$.

\section{RESULTADOS}

Analizados los resultados de observacion del comportamiento de los estudiantes en el aprendizaje y conceptualización en temas de geometria y de la encuesta aplicada los mismos, se observa que el $68 \%$ de los estudiantes manifiestan que aunque "entienden" los conceptos geométricos, tienen dificultad para identificarlos en su entorno. Ademas la mayoria argumenta tener dudas e inquietudes referentes a algunos temas, pero que por cuestiones de temor a preguntar prefieren quedarse con las mismas. El $100 \%$ de los estudiantes tienen mascotas en sus viviendas, y dentro de estas es infaltable el perro, por sus ventajas y utilidad bien conocidas por todos,al ser pregntados si les gustaria reforzar sus conocimientos de geometría utilizando su mascota, el $100 \%$ contestó que si, que seria muy interesante y novedoso.

El diálogo con el maestro permite identificar apreciaciones de los estudiantes se propone la aplicación de la metodologia de aula invertida y utilizar a la mascota preferida por los niños como herramienta pedagogica, para la explicacion y mecanizacion de los conceptos de la geometria.

\subsection{Propuesta didáctica}

De acuerdo a los estándares del Ministerio de Educación Nacional, se espera que los estudiantes en grado quinto identifiquen $y$ clasifiquen las figuras geométricas planas reconociendo algunas de sus propiedades básicas. La propuesta involucra objetivos, competencias y resultados de aprendizaje en grados de quinto a undécimo, a fin de que los estudiantes conceptualicen, generen ideas, analicen, interpreten por si mismos por medio de actividades guiadas por los maestros, otorgándoles un aprendizaje más significativo. En torno a actividades a realizar, a partir de algunas propuestas realizadas por estudiantes 0 sus padres y otras planteadas por el maestro, se organizan temas en geometría lineal, geometría euclidiana, geometría diferencial, cálculo infinitesimal, modelado e inicio de ecuaciones diferenciales (Tabla 1).

La metodología en la propuesta pedagógica utilizada es clave en el proceso de aprendizajeenseñanza, por lo tanto, es necesario hacer uso de una metodología didáctica, un plan estructurado que puede usarse para diseñar materiales de enseñanza y orientar esa enseñanza en las aulas (Romero, Torres, Vergel, 2020). En ese sentido, se propone la puesta en práctica de una serie de actividades en el último curso de educación básica del modelo Escuela Nueva. Se sugiere el implementar la metodología de trabajo colaborativo con el modelo Flipped Class Room (clase invertida), se propone trabajar como un gran equipo equitativo de trabajo escolar para alcanzar objetivos y metas comunes que de forma individual sería imposible. Lo anterior convierte a los estudiantes en protagonistas de su propio aprendizaje.

Se trabaja en pequeños grupos, en los que juntos guían su propio aprendizaje y el de los demás, socializando los resultados de las actividades realizadas de forma individual con ayuda de su mascota, el proceso se realiza con 
la orientación y guía del maestro. Este método de aprendizaje permite el intercambio de conocimientos, ideas, concretar conocimientos, identificar líderes, perder el miedo a expresar las dudas y a fortalecer valores y la amistad. Los contenidos son seleccionados de acuerdo a las temáticas registradas en el plan de estudios para la asignatura de geometría, diseñados en su mayoría en las guías de escuela nueva, el cual cumple con los estándares necesarios de aprendizaje que se exigen desde el Ministerio de Educación Nacional, y se vinculan nuevas temáticas acorde a actividades que propongan os estudiantes y se reincorporan en el plan de actividades y desarrollo de competencias y habilidades.

Crooks y Alibali, (2014) plantean que "el pensamiento geométrico en el niño de Educación Infantil se construye de forma progresiva, como corresponde a las características generales de su pensamiento. Además, los conceptos geométricos son elaborados por el niño fundamentalmente a partir de la percepción de sí mismo y de lo que le es próximo". Con el fin de proponer una clase más motivante para los estudiantes, se establece una relación entre los conceptos de geometría y la recolección de información con el uso de cada mascota (perro) buscando sus demostraciones más prácticas, creativas, innovadoras dando igual importancia a cada uno de los contenidos en los ejes temáticos.
Se propone incluir actividades didácticas que permitan fortalecer el aprendizaje de los diferentes conceptos, mediante guías divididas por temas de aprendizaje que incluyen objetivos, listado de conceptos previos a tener en cuenta en el desarrollo de la guía y las actividades a desarrollar, formulando además preguntas con diferentes grados de dificultad que permitan al estudiante llegar al concepto o propiedad requerida.

Después de implementar lo recomendado, se observó el rendimiento (Tabla 1) y se evaluó la asimilación de los temas de geometría, orientados bajo la nueva metodología, el resultado encontrado evidencia que los niños comprendieron mejor y de forma divertida los conceptos impartidos toda vez, que en sus hogares junto a sus mascotas estudiaron los temas a través de las guías impartidas por los maestros y luego socializaron y aclararon las dudas e inquietudes que se les presentaron.

Análisis conceptual permite identificar categorías emergentes en temas y actividades desarrolladas en diferentes grados (Tabla 1). Así mismo, los resultados obtenidos antes y después de la intervención educativa se exponen en la tabla 1. Además de diferenciar cambios en la actitud, motivación por el aprendizaje, generación de ideas, se observan cambios en conocimiento general sobre geometría a través de las mascotas caninos y conocimientos sobre su evolución, movimiento y cuerpo. 
Tabla 1. Categorías emergentes - resultados pretest y post-test

\begin{tabular}{|c|c|c|c|c|c|}
\hline Tipo & Concepto & Actividad didáctica & $\begin{array}{c}\text { promedio- } \\
\text { pretest }\end{array}$ & $\begin{array}{c}\text { promedio- } \\
\text { postest }\end{array}$ & $p$ \\
\hline Geometría lineal & Línea & Dibujo & 45 & 95 & 0.001 \\
\hline $\begin{array}{l}\text { Geometría } \\
\text { Euclidiana }\end{array}$ & $\begin{array}{l}\text { Triangulo, } \\
\text { cuadrado, } \\
\text { rectángulo, } \\
\text { circunferencia, } \\
\text { razón aurea } \\
\text { Áreas }\end{array}$ & $\begin{array}{l}\text { Dibujo } \\
\text { Arte: Cuadros } \\
\text { madera y cartón } \\
\text { Arte Origami } \\
\text { Tangram } \\
\text { Composiciones- } \\
\text { Mosaicos } \\
\text { Pintura libre óleo, } \\
\text { lápiz }\end{array}$ & 57 & 91 & 0.005 \\
\hline $\begin{array}{l}\text { Geometría } \\
\text { Diferencial }\end{array}$ & $\begin{array}{l}\text { Ángulos, razones } \\
\text { trigonométricas }\end{array}$ & $\begin{array}{l}\text { Dibujo } \\
\text { Proyección } \\
\text { Medición real en } \\
\text { ser vivo } \\
\text { Video análisis }\end{array}$ & 62 & 92 & 0 \\
\hline $\begin{array}{l}\text { Cálculo: } \\
\text { Relaciones y } \\
\text { Funciones } \\
\text { Variacional }\end{array}$ & $\begin{array}{l}\text { Plano cartesiano } \\
\text { Línea } \\
\text { Cónicas } \\
\text { Rectas paralelas } \\
\text {-perpendiculares } \\
\text { Velocidad } \\
\text { Funciones } \\
\text { Razón de cambio } \\
\text { derivadas }\end{array}$ & $\begin{array}{l}\text { Proyecciones } \\
\text { Geogebra }\end{array}$ & 51 & 90 & 0.001 \\
\hline
\end{tabular}




\begin{tabular}{|l|l|l|l|l|l|}
\hline Modelado & Derivadas & Autocad & 43 & 98 & 0.003 \\
& Área Volumen & Geogebra & & \\
Transformaciones & $\begin{array}{l}\text { Construcción } \\
\text { prototipos prótesis } \\
\text { en diferente } \\
\text { material } \\
\text { pruebas }\end{array}$ & & & \\
\hline
\end{tabular}

Fuente: Autores

Al igual que Portero, Cirne y Mathiu (2002) la metodología en cada encuentro con niños y jóvenes, procesos en torno a lo informativo y formativo se basan en demanda asociada a la coherencia donde existe correlación positiva entre concepciones e institución similar a resultados en creatividad de Nieto, Rojas y Vergel (2019), constancia asociada a frecuencia de actividades realizadas por los niños (superior a 5 en la semana dada alta actividad con las mascotas) y, la extensión, relacionada con acciones de inclusión. Resultados permiten apreciar cambios significativos a un nivel de confiabilidad de $95 \%$ en pro de la mejora del rendimiento de los estudiantes en geometría.

\section{CONCLUSIONES}

La estrategia didáctica a través del estudio de características de las mascotas, permite al niño relacionarse con elementos de su entorno y ser el artífice de la construcción de su conocimiento, además de diferenciar cambios en la actitud, motivación por el aprendizaje, generación de ideas, se observan cambios en conocimiento general sobre geometría a través de las mascotas caninos y conocimientos sobre su evolución, movimiento y cuerpo.

El método de aula invertida apoyado en la interacción con las mascotas (perro), mejora el interés por el aprendizaje de la geometría, estimula la aplicación de las matemáticas en la vida cotidiana, genera curiosidad y creatividad, fortalece el trabajo en equipo, crea espacios democráticos donde se respeta la opinión de los compañeros, creando en los estudiantes una relación de conceptos geométricos bien definidos que le permitan entender el mundo desde lo medible, proporcionar un cambio en el uso de estos conceptos en otras áreas de conocimiento como lo es el arte y de esta forma lograr que observen el mundo desde lo estético y lo simétrico.

Categorías emergentes en geometría fueron geometría lineal, geometría euclidiana, geometría diferencial, cálculo: relaciones y funciones, variacional, modelado; subcategorías asociadas a temas en Línea, triángulo, cuadrado, rectángulo, circunferencia, razón aurea, áreas, ángulos, razones trigonométricas, plano cartesiano, cónicas ,rectas paralelas, perpendiculares, velocidad, funciones, razón de cambio, derivadas, volumen, transformaciones.

Resultados permiten apreciar cambios significativos a un nivel de confiabilidad de $95 \%$ en pro de la mejora del rendimiento de los estudiantes en geometría

\section{REFERENCIAS BIBLIOGRÁFICAS}

Andrew, T. B., \& Edney, B. A. (1995). Companion animals and human health: an overview. Journal of the Royal Society of Medicine, 88, 704-708. Recuperado de http:// europepmc.org/backend/ptpmcrender. $\underline{\text { fcgi?accid=PMC1295422\&blobtype }=\text { pdf }}$ 
Correa Duque, María Cristina. (2019). Educación asistida con perros: aplicaciones pedagógicas en contextos educativos

Crooks, N. y Alibali, M. W. (2014). Defining and measuring conceptual knowledge in mathematics. Developmental Review, 34 (4), 344-377

Gómez CS, Rojas JP y Vergel M 2020 Estrategia gerencial seis sigma en la gestión de la vicerrectoría de bienestar universitario. (Bogotá: ECOE ediciones)

Godino Juan D (2003) Matemáticas y su didáctica para maestros. Facultad de Ciencias de la Educación Universidad de Granada. Granada

Hernández R Fernández $C$ and Baptista P 2010 Metodología de la investigación Mc Graw Hill

Lage, M. J., Platt, G. J., \& Treglia, M. (2000). Inverting the classroom: A gateway to creating an inclusive learning environment. The Journal of Economic Education, 31(1), 30-43.

Latorre, Antonio (2003), La investigación-acción: Conocer y cambiar la práctica educativa. España, GRAÓ

Méndez-Molina B L, Moreno L y Vergel-Ortega M 2019 Infección de aislados nativos de Burkholderia glumae en variedades de semillas de arroz certificadas (Bogotá: ECOE Ediciones)

Maldonado H, Vergel-Ortega, M y Rojas-Suárez J P 2020 Innovación y creatividad: una estrategia pedagógica en la enseñabilidad del cálculo aplicado a la física electromagnética (Bogotá: Ecoe ediciones)
Nieto Contreras J F, Rojas-Suarez J P y VergelOrtega M 2019 Impacto de estrategia pedagógica basada en el aprendizaje creativo para estudiantes de ingeniería (Bogotá: Ecoe Ediciones)

Portero López P, Cirne Lima R, Mathieu G. La intervención en adolescentes y jóvenes en la prevención y promoción de la salud. Rev. Esp. Salud Pública 2002; 76:577-584.

Romero E, Torres JM y Vergel M 2020 Representaciones sociales de estudiantes universitarios sobre el aprendizaje del cálculo y la química (Bogotá: ECOE Ediciones)

Tonucci Francesco, (2008). Recuperado el 10 de Diciembre de 1020 de https://www. lanacion.com.ar/cultura/la-mision-principalde-la-escuela-ya-no-es-ensenar-cosasnid1085047/

Sams, A., \& Bergmann, J. (2012). Flip your classroom: Reach every student in every class every day. International Society for Technology in Education (ISTE). 